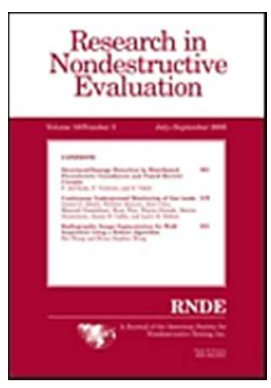

\title{
Automated quality characterization for composites using hybrid ultrasonic imaging techniques
}

\begin{tabular}{|r|l|}
\hline Journal: & Research in Nondestructive Evaluation \\
\hline Manuscript ID & URND-2017-0052.R2 \\
\hline Danuscript Type: & Original Paper \\
\hline Complete List of Authors: & $\begin{array}{l}\text { Sun, Jiangtao ; Brunel University London, Brunel Innovation Centre } \\
\text { Chong, Alvin ; Brunel University London, Brunel Innovation Centre } \\
\text { Tavakoli, Siamak ; Brunel University London, Brunel Innovation Centre } \\
\text { Feng, Guojin; Brunel University London, Brunel Innovation Centre } \\
\text { Kanfoud, Jamil; Brunel University London, Brunel Innovation Centre } \\
\text { Selcuk, Cem; Brunel University London, Brunel Innovation Centre } \\
\text { Gan, Tat-Hean; Brunel University, Brunel Innovation Centre }\end{array}$ \\
\hline Keywords: & $\begin{array}{l}\text { Carbon-fibre-reinforced polymer, Glass-fibre-reinforced polymer, ultrasonic } \\
\text { testing, image processing }\end{array}$ \\
\hline &
\end{tabular}




\title{
1 Automated quality characterization for composites using
}

\section{2 hybrid ultrasonic imaging techniques}

\author{
3 Jiangtao Sun ${ }^{1}$, Alvin Yung Boon Chong ${ }^{1}$, Siamak Tavakoli ${ }^{1}$, Guojin Feng ${ }^{1}$, Jamil Kanfoud ${ }^{1}$, Cem \\ 4 Selcuk $^{1}$ and Tat-Hean Gan $^{1, *}$ \\ $5{ }^{1}$ Brunel Innovation Centre (Brunel University London), Granta Park, Great Abington, Cambridge, \\ $6 \quad$ CB21 6AL, United Kingdom. \\ $7 \quad$ *Corresponding author at: email: tat-hean.gan@brunel.ac.uk, Tel: +44 (0)1223 899455 \\ 8
}

9 Abstract

10 An enhanced technique using image processing has been developed for automated ultrasonic

11 inspection of composite materials, such as glass/carbon-fibre-reinforced polymer (GFRP or CFRP), to

12 ascertain their structural healthiness. The proposed technique is capable of identifying the abnormality

13 features buried in the composite by image filtering and segmentation applied to ultrasonic C-Scan

14 images. This work presents results performed on two composite samples with simulated delamination

15 defects. A local gating scheme is applied to raw A-Scan data for improved contrast between defective

16 and healthy regions in the produced C-Scan image. In this test campaign, different filtering and

17 thresholding algorithms are evaluated and compared in term of their effectiveness on defect

18 identification. The accuracies of less than $3 \mathrm{~mm}$ and $1.11 \mathrm{~mm}$ were attained for the defect size and

19 depth respectively. The results demonstrates the applicability of the proposed technique for accurate

20 defect localization and characterization of composite materials.

21 Keywords: Carbon-fibre-reinforced polymer, Glass-fibre-reinforced polymer, ultrasonic testing, 22 image processing. 


\section{1. Introduction}

2

3 It is widely known that composite materials bring tremendous benefits, such as high stiffness to

4 density ratio and high strength to weight ratio, which are not available in traditional materials. Due to

5 their superior properties, the use of composites as structural components has been gradually

6 increasing in aerospace and energy (wind turbines). From the aerospace perspective, the average

7 usage of composite by weight has increased more than 7 fold in a span of around 3 decades (i.e. an

8 average of $7 \%$ for Airbus in 1980 to $53 \%$ in Airbus 350 XWB series) [1]. However, the manufacture

9 process for fibre reinforced composites has the potential to introduce a variety of processing defects,

10 e.g. delamination, discrete void and porosity [2]. These defects can lead to delamination between

11 layers or within fibre matrix either during the manufacturing process or after the component enters

12 service, e.g. the component may be accidentally impacted by external forces during its service period.

13 As a common type of defect in composites, delamination is the results of layer separation caused by

14 the loss of internal bonding between adjacent layers or layer groups. As a severe mechanical

15 discontinuity, it fails in the transfer of inter-laminar shear stresses, which leads to rapid and

16 catastrophic buckling failure when large compressive load or force is imposed on it [3, 4]. This brings

17 the necessities for early-stage delamination detection and characterisations to prevent potential

18 failures of composite parts, which will be the main topic of this paper. There are a variety of

19 inspection methods available for composite materials, e.g. infrared thermography, ultrasonic testing

20 (UT) technique, acoustic emission testing, optical, laser technique, microwave testing, X-ray

21 computed tomography (CT), eddy current techniques and acid digestion. Almost all of those test

22 methods are non-destructive testing (NDT), except for the acid digestion method (destructive). UT

23 can be easily and effectively used for quality control and materials quality inspection in all major

24 industries [5]. Most of other methods, however, are either still at their research stages or have

25 limitations on their wide applications due to cost, detection reliability, portability, equipment setup,

26 scanning time and safety concerns. UT is considered as the most favourable choice for composite

27 inspection, either at the manufacturing or maintenance stages. Also because of its high sensitivities to 
1 delamination in the material ply or porosity which are the most common types of defects found in

2 composites [6].

3 Ultrasonic testing methods emit stress waves into the material or component to be inspected and then

4 the transmitted or reflected signals is measured using a separate transducer on the opposite surface of

5 the material or using the same transducer for signal excitation and acquisition, namely through-

6 transmission or pulse-echo technique, respectively [7]. The through-transmission technique requires

7 the two transducers to be installed on the opposite sides of the material under inspection, which may

8 not be practical in many applications. It also cannot provide the depth information of the defects,

9 while the pulse-echo technique can give this information by detecting the arrival time or time of flight

10 (TOF) of ultrasonic echoes reflected from a defect within a test component. Therefore, the research

11 scope of this work focuses on the pulse-echo technique. A-scan data is acquired at each scan position

12 in the composite, which gives the amplitude of received ultrasonic reflection signal against its TOF. A

13 C-scan image is then generated by converting the A-scan raw data at each scan position into a pixel

14 value through appropriate gating and thresholding, which results in a superimposed planar view of the

15 test piece with defect location and size. Ultrasonic C-scan imaging technique has been exploited in

16 many applications for composite inspection. A few examples include characterization of artificial

17 delamination [8], detection of impact damage in carbon or epoxy composite plates [9],

18 characterization of void distribution, size and shape in composites [10] and extraction of special

19 features of the interfaces between fibres or matrix [11]. To determine the defect sizes in composite

20 materials, guidelines were suggested for the UT inspection which concluded that the efficient

21 estimation of defect size is difficult to be achieved, especially in composites with multiple layers and

22 a large thickness $[5,13]$. Hasiotis et al. located several artificial delamination, which were simulated

23 by embedding 'Upilex', a high heat resistance polyamide material into multiple-layer composite

24 plates, through appropriate tuning of a commercially available UT inspection system. The specimen

25 thickness and the depth position of the defects can be determined with their shapes and sizes attained

26 in some cases. 
1 Currently, NDT inspection of composites in manufacture and service is still largely manual with low

2 area coverage. In addition, due to macroscopic structural anisotropy which produces high noise levels

3 [14], the NDT images are difficult to interpret quantitatively for any technique, including the most

4 popular UT inspection. Therefore, the appropriate collection and interpretation of UT data highly

5 depends on the experience of an operator, which is slow and not always reliable. Smith et al. [15]

6 discussed the necessities to develop a technique for automated analysis and advanced defect

7 characterisation from UT scans of composites for aerospace applications. The reasons include (i) the

8 large amount of data to be analysed, i.e. a Terabyte of data per airliner and over 200 Terabytes per

9 year internationally, (ii) the focus of operator's attention on the inspection, (iii) analysis of faulty composite components and (iv) the acquisition of 3D quantitative information about the defects to facilitate final defect recognition process via decision-making by the operator. It was pointed out that

12 conventional analysis methods for UT inspection can give very limited information for the stress 13 analysis of defects due to the average effect in thickness direction of the materials, while 3D advanced 14 characterization can resolve this issue and assist in defect detection during the disposition process of composites. An automated methodology using reference scan was also proposed to compare the reference data with acquired data for the same component. The attained differences after comparison

17 were then classified according to their causes, i.e. noises, misalignment or defects, with useful 18 features of the identified defects measured for a 'good' or 'faulty' decision-making against the manufacturer's acceptance criterion. This automated process may be alternatively accomplished by a dedicated image segmentation and defect recognition process, which offers the advantage of omitting the usage of reference image described in this study. Thus, an automated UT defect characterization

22 technique is proposed to identify and recognize a number of artificial defects in test samples, made of polytetrafluoroethylene (PTFE) tapes. Two types of composites, i.e. GFRP and CFRP, were studied to validate the feasibility of the test method on different material types for effective inspection in each case. The defects are buried into multi-layer test laminates, for both GFRP and CFRP, to simulate typical delamination. Each test sample has multiple defects, varying in their sizes and depth positions

27 for systemic evaluation. For defect identification, the outline design of the automated UT inspection 28 method is discussed in particular the dedicated image processing steps such as image filtering, 
1

2

3

4

5

6

7

8

9

1 segmentation and 3D characterization including 3D locations and sizes of defects. The inspection

2 results are shown and analysed for a curved GFRP sample and a flat CFRP sample with simulated

3 defects.

\section{2. Experimental setup}

\section{$5 \quad$ 2.1. Test sample preparation}

6 In this study, two test samples, (i) curved GFRP and (ii) flat CFRP, were prepared using vacuum7 bagging process [16]. The properties of the curved GFRP sample with defects can be found in Table 1 .

8 A total of 16 simulated defects were introduced by inserting round pieces of PTFE tapes, placed 9 between layers and at different depths. Those defects approximately make a $4 \times 4$ matrix, with varying sizes in each column and varying depths in each row.

11 The flat CFRP sample is around $3.6 \mathrm{~mm}$ in thickness and contains 16 simulated PTFE defects 12 arranged as a $4 \times 4$ matrix, as shown in Fig. 2. PTFE defects are adopted because they are easy to 13 manufacture (embedded between adjacent laminate layers) and have smaller contrast to the composite 14 material than air (i.e. where delamination occur) in term of ultrasonic attenuations. This means that 15 delamination can be detected if the PTFE defects are successfully identified. Similarly, the 4 defects 16 in each row have identical sizes but are located at different depths inside the sample, while the 4 17 defects in each column were located at the same depth but of different sizes. The characteristics of the defects are shown in Table 2, i.e. the size (the diameter of each circular defect) and depth of each 19 defect along the height of the sample. It is worth noting that the 4 defects in each row or column were not exactly aligned with each other in practice due to the influences of the manufacturing process.

\subsection{Manual inspection system setup}

24 There are 2 UT experimental setups used in this study. The first setup is for manual inspection of the 25 GFRP sample due to its curvature and size. The second setup is for automated inspection using 
1 conventional immersion tank setup for flat samples. The manual setup consists of four parts and is

2 shown in Fig. 3:

3 a. Pulse transmitter and receiver: Topaz (using conventional ultrasonic testing mode) [17];

4 b. UT probe: $5 \mathrm{MHz}$ single crystal compression probe;

5 c. Wedge: Custom Perspex wedge (10 mm thick);

6 d. Encoder: String pot.

7 In the experiment, the ultrasonic inspection was done using contact transducers with coupling being

8 achieved by applying gel to the surface of the curved composite. Due to the thickness of the

9 composite sample (ca. $4 \mathrm{~mm}$ ), a wedge is placed between the ultrasonic sensor and the composite to

10 separate the defect reflection signals from the input signal (i.e. effectively a delay line). The ultrasonic

11 probe was adhered to the wedge and attached to the string pot encoder to determine the scan position.

12 The reflection or echo signal at each scan position on the surface of the GFRP sample was recorded in

13 A-Scan format. The scan setting is shown in Table 3 which shows that each scan is taken along the 14 scan axis due to that the scan is made manually with a 1D encoder (i.e. a single straight line scan).

15 The scan axis is approximately parallel to the column direction of the $4 \times 4$ defect matrix, as shown in 16 Fig. 1. At each scan position, 1092 sampling values of the UT reflection signal are recorded with a 17 depth resolution of $0.03 \mathrm{~mm}$, which gives the length of the A-Scan data at each position.

\subsection{Automated inspection setup}

Automated UT scanning is performed using water immersion inspection as shown in Fig. 4 for the 21 experimental setup of flat CFRP sample with PTFE defects. Rectangle metal blocks were used as supporting structure and the setup ensure the composite surface is level to the probe face. The standoff distance between the sample and the water tank is ca. $37 \mathrm{~mm}$. The standoff distance between the UT 24 probe and the sample was varied for optimised inspection, e.g. $70 \mathrm{~mm}$ or $130 \mathrm{~mm}$. At each scan 25 position, 1348 sampling values of the UT reflection signal are recorded with a depth resolution of $260.03 \mathrm{~mm}$, which gives the length of the A-scan data at each position. The excitation frequency of the 27 UT probe was chosen to be $5 \mathrm{MHz}$ and $10 \mathrm{MHz}$ for comparison purpose. The sampling rate of the 
1 system is $100 \mathrm{MHz}$. The scan setting with the excitation frequency of $5 \mathrm{MHz}$ is shown in Table 4. The

2 automatic scan covers the whole surface of the test piece with margin extended around each edge of

3 the sample.

4

\section{3. Image processing for ultrasonic C-Scan images}

6

\section{$7 \quad$ 3.1. Image enhancement}

9 One of the critical criteria for automated defect recognition is to apply appropriate techniques for 10 image segmentation since all subsequent quantitative analysis depends on it. 'Binarization' process 11 (converting a pixel image to a binary image) is usually required to segment images into two parts, i.e.

12 foreground defects and background materials, which results in a morphological representation of the 13 composite materials to determine the characteristics of defects such as porosity and delamination.

14 Within the framework of segmentation, it is necessary to include image enhancement due to the high 15 noise levels produced in the UT A-Scan for composite. In addition to anisotropy effects, unstable 16 contact coupling between the UT probe and the composite sample may introduce additional noises.

17 Consequently, poor results would mostly be attained if thresholding techniques for 'binarization' are 18 applied directly. This problem can be partially alleviated by pre-processing the C-scan images with 19 de-noising filters described in section 3.2. A post-processing can also be applied to the binary image 20 after segmentation to further alleviate the effects of high level noises using morphological operations 21 such as erosion and dilation [18]. Segmentation quality, however, would be reduced by excessive pre22 processing or post-processing since the processing would indiscriminately eliminate not only noises 23 but also true defects and may generate undesired artefacts. Therefore, the use of filtering or 24 morphological operations for noise removal should be made with care (i.e. appropriate filter size 25 selected for image pre-processing).

\subsection{Image filtering}


2 Filtering is a crucial step prior to image segmentation because images are usually contaminated by

3 noise, e.g. Gaussian noise, 'salt-pepper' noise and digital quantization noise and direct segmentation

4 without filtering would be erroneous. Different filters, i.e. linear or non-linear and in spatial or

5 frequency domains are applied on different images based on their noise types for noise removal or

6 smoothing. Filters operating in spatial domain are convolved with the images to be processed for

7 noise removal, while filters operating in frequency domain are simply multiplied with the frequency

8 transforms of the images to be processed. In an image, noise contamination can be modelled in an

9 additive manner as follows [19]:

$11 \quad g(x, y)=f(x, y)+n(x, y)$

13 where $g(x, y)$ is the contaminated image in the first equation, $x$ and $y$ are the two dimensional (2D) 14 coordinates in spatial-domain, $f(x, y)$ is the original (noise-free) image and $n(x, y)$ represents the 15 image noise. In this study, spatial domain filters are adopted for noise removal in UT C-scan images 16 based on their suitability. A-scan data of composites are essentially noisy because of multiple 17 scattering from the fibre-matrix boundaries which is always present, with or without defects. This 18 noise makes defect detection difficult especially when the defects are small. This is because the 19 'useful' signal would be at almost the same level as the noises in this case. The gating operation will 20 produce a C-scan image with small defects overwhelmed by noises and direct segmentation will be 21 faulty with lots of false 'alarms'. Through experiments, it is found that median filter and Gaussian 22 low-pass filter can be applied prior to segmentation to alleviate this issue. An example of a 2D median 23 filter with a $3 \times 3$ neighbourhood window is shown in Fig. 5. The median value within the window is 24 assigned to the central pixel, i.e. using 60 to replace the extreme value 255 of the central pixel which 25 may be randomly occurred due to speckle noise. 
1 Alternatively, Gaussian low-pass filter is utilized for high frequency noise removal which highest

2 weight is assigned to the centre pixel and the weight value reduces with increase in pixel distance

3 from the centre. A typical 2D Gaussian distribution function can be defined as:

4

$5 \quad G(x, y)=\frac{1}{2 \pi \sigma^{2}} \exp ^{-\frac{x^{2}+y^{2}}{2 \sigma^{2}}}$

6 This distribution assumes zero means in both directions (i.e. $\mu_{x}=\mu_{y}=0$ ) and standard deviations 7 of $\sigma_{x}=\sigma_{y}=\sigma$, and a zero covariance between the distributions in both directions (i.e. $\sigma_{x y}=0$ ). Its 8 graphical representation with $\sigma=1$ is shown in Fig. 6. Subsequently, the Gaussian function needs to 9 be discretized or sampled to produce a specified set of Gaussian kernel coefficients, where the size of 10 which is limited to contain only sampling values within three standard deviations from the mean of 11 the Gaussian function since $99 \%$ of the distribution falls within this range. The kernel coefficients 12 depend on the value of $\sigma$ and diminish with increasing distance from the kernel's centre. The larger 13 the $\sigma$, the larger the kernel size needs to be to maintain the Gaussian nature, which produces a more 14 blurred image after filtering.

\subsection{Image segmentation based on thresholding}

Defects in C-Scan image can be characterized, e.g. their sizes, locations and shapes, by segmenting the image into its constitutive parts, mostly the object foreground and the background, which can be regarded as a two-class classification problem. The less percentage of misclassification of the two classes (i.e. the error rate) is, the better the segmentation technique. Therefore, minimization of this error rate is the target of all segmentation methods. In general, segmentation techniques can be classified into four different categories [20-23]: (1) straightforward histogram-based techniques (e.g. global and local thresholding), (2) region-based techniques (e.g. region growing and watershed), (3)

25 pixel classification techniques (e.g. fuzzy c-means, Markov random fields, artificial neural networks)

26 and (4) model-based techniques (e.g. parametric and geometric deformable models or level sets).

27 Despite of the attained benefits, those techniques except for the thresholding techniques, are usually 
1 complex and require advanced mathematical development and several pre- or post-processing steps 2 [24]. On the other hand, the thresholding techniques, despite some of them are from the 80 's, are still

3 very popular because they are simple to understand and implement, and computationally efficient 4 once the histogram has been obtained. Additionally, a considerable part of them require minimal 5 human efforts to determine the threshold [25], which enables automated thresholding and 6 subsequently automated defect detection. Therefore, thresholding techniques are adopted for image 7 segmentation in this study $[25,26]$. Two-level thresholding can be expressed as:

\section{8}

$9 g(x, y)= \begin{cases}1 & \text { if } f(x, y)>T \\ 0 & \text { if } f(x, y) \leq T\end{cases}$

11 where $f(x, y)$ is the original image, $T$ is the threshold value and $g(x, y)$ is the thresholded binary 12 image. Thresholding algorithms compute a threshold $T$ based on a given distribution or histogram of 13 brightness or intensity levels.

14 Automated thresholding algorithms [26] are used in our application since they can adapt to different 15 ultrasonic C-scan imaging scenarios with minimal user interventions. Four algorithms namely (i) 16 moments-based, (ii) entropy-based, (iii) Ostu's and (iv) minimum error methods are empirically 17 chosen for performance comparison in this study since they can produce acceptable segmentation 18 results. The histogram of an image can be denoted as $h_{0}, h_{1}, h_{2}, \ldots, h_{n}$, where $h_{j}$ is the number of 19 pixels with intensity level $j$ in the image, and $n$ is the maximum intensity level in the image which is 20255 for an 8-bit grey image. The threshold value $T$ is an integer calculated based on this histogram 21 and used as the criterion for the classification of all the pixels into two categories, i.e. foreground and 22 background. To describe the thresholding algorithms adopted in this study, the following partial sums 23 are defined:

$$
A_{j}=\sum_{i=0}^{j} h_{i} \quad B_{j}=\sum_{i=0}^{j} i h_{i} \quad C_{j}=\sum_{i=0}^{j} i^{2} h_{i} \quad D_{j}=\sum_{i=0}^{j} i^{3} h_{i}, \quad j=0,1, \ldots, n
$$


1 Tsai [27] proposed an algorithm for image thresholding by choosing a threshold $T$ such that the

2 segmented image has preserved the first three moments of the original grey image (known as

3 moments-based method). This is achieved by setting $T$ such that $A_{T} / A_{n}$ is the value of the fraction

4 nearest to $x_{0}$, where:

5

$6 \quad x_{0}=\frac{1}{2}-\frac{B_{n} / A_{n}+x_{2} / 2}{\sqrt{x_{2}^{2}-4 x_{1}}}, \quad x_{1}=\frac{B_{n} D_{n-} C_{n}^{2}}{A_{n} C_{n}-B_{n}^{2}}, \quad x_{2}=\frac{B_{n} C_{n-} A_{n} D_{n}}{A_{n} C_{n}-B_{n}^{2}}$

7

8 Kapur et al. [28] introduced an entropy-based thresholding method. The threshold $T$ is set to the 9 intensity level $j$ at which the entropy formulation,

10

$11 \frac{E_{j}}{A_{j}}-\log A_{j}+\frac{E_{n}-E_{j}}{A_{n}-A_{j}}-\log \left(A_{n}-A_{j}\right)$

12

13 is maximized for $j=0,1, \ldots, n-1$, where

$15 E_{j}=\sum_{i=0}^{j} h_{i} \log h_{i}, j=0,1, \ldots, n$

17 Otsu [29] chose the threshold $T$ by maximizing the between-class variance of foreground and 18 background in an image (known as Otsu's method):

$A_{j}\left(A_{n}-A_{j}\right)\left(\mu_{j}-v_{j}\right)^{2}, j=0,1, \ldots, n$

21

22 where

23

$24 \quad \mu_{j}=\frac{B_{j}}{A_{j}}, v_{j}=\frac{B_{n}-B_{j}}{A_{n}-A_{j}}$

25 
1 In which the threshold $T$ estimated using the Otsu's method lies in the midway between the means of

2 the two classes (foreground and background).

3 Kittler and Illingworth [30] assumed a Gaussian mixture model for the pixels in the two classes of an

4 image with the image histogram being the probability density function of the mixture population.

5 Several statistics are defined for estimating the threshold:

6

$7 \quad p_{j}=\frac{A_{j}}{A_{n}}, q_{j}=\frac{A_{n}-A_{j}}{A_{n}}, \sigma_{j}^{2}=\frac{C_{j}}{A_{j}}-\mu_{j}^{2}, \tau_{j}^{2}=\frac{C_{n}-C_{j}}{A_{n}-A_{j}}-v_{j}^{2}$

8

9 The threshold $T$ is derived by minimizing (referred to as minimum error algorithm)

10

11

$p_{j} \log \frac{\sigma_{j}}{p_{j}}+q_{j} \log \frac{\tau_{j}}{q_{j}}, j=0,1, \ldots, n$

12

13 The selected algorithms were implemented in Matlab 2014a for performance evaluation in term of 14 defect identification capability in C-scan images of composite samples under test. Besides applying a 15 single method for thresholding, hybrid methods can also be used for improved performance. For 16 example, two methods may be complementary, i.e. one method underestimates the threshold while the 17 other overestimate the threshold, and the average of those two thresholds may give a more accurate one. This will be discussed and proved with experimental results in section 4.2.3.

\subsection{Morphological operation}

21 As a post-processing technique, mathematical morphology can be used to analyse and process 22 geometrical structures or extract useful image components to represent and describe the shape of a 23 region, e.g. its boundaries and the convex hull [19]. On the basis of set theory, simple mathematical 24 operators are derived for morphology, which can be exploited to remove noise, enhance image, and 25 detect object edges in binary images. They can also be applied to grey-scale images, but not as widely 26 used as in binary images since they are simpler and more efficient on binary images. Typical 
1 morphological operators include dilation and erosion, opening and closing, hit and miss operation,

2 thinning and thickening. Dilation and erosion are two basic morphological operations [31]. Dilatations

3 and erosions are usually utilised in pairs to constitute morphological opening and closing. Opening by

4 a specific-shaped structuring element can smooth the boundary, breaks narrow parts, and eliminates

5 small objects. Closing by a specific-shaped structuring element can smooth the boundary, fills narrow

6 bays, and eliminates small holes. Based on those basic operations, there is an operation for binary

7 images based on the areas of connected image components (objects), i.e. the area opening to remove

8 all connected components that have less than a certain number of pixels (the minimum object size to

9 be detected defined by users) from the binary image. In view of our purpose in this study, i.e.

10 automated post-filtering of the binary image after thresholding to remove small residual spots due to

11 noise in the original $\mathrm{C}$-scan image, it is appropriate to define the required detection resolution by

12 using the area opening operation with the minimum object size (in pixels) to be detected.

\subsection{Characterization of defect features}

14 After the morphological operation, a 'clean' binary image can be obtained in which defect regions can be labelled and characterized. All the pixels belonging to the same defected region are labelled with a single integer number. Then the size of each defect can be determined easily by counting the number

17 of pixels with the corresponding label and its location can be derived by calculating the centroid of these pixels. The characterization results can be used for eliminating the false defects due to the loss of coupling at the beginning or end of the ultrasonic scanning, which can be identified by the defect location.

\section{4. Results and discussion}

\subsection{C-Scan imaging of GFRP sample and processing results}

25 The experimental setup for the GFRP sample has been described with details in section 2.2, i.e. 
1 especially when defects are small and UT coupling is not sufficiently good. Therefore, it is not

2 reliable to use the typical gating between the front and back wall of the composite sample to identify

3 possible defects. A double gating scheme is proposed, with the first gating to generate a C-Scan image

4 and the second gating to derive the depth of detected defects. The first gating is placed around the

5 back wall reflection of the composite sample, as shown below in Fig. 8, because it was observed that 6 significant amplitude attenuation of UT reflection occurs in this region when a defect is present. This 7 gating is used to generate a C-Scan image from the corresponding A-Scan data for each UT scan.

8 With the C-Scan image, it is viable to segment the possible defects from the image background by 9 applying appropriate thresholding techniques after image filtering and determine the centres of defects 10 (in term of $\mathrm{X}$ and $\mathrm{Y}$ index in the UT scanning matrix). The raw A-Scan data at the located centre for 11 each defect was used to estimate the defect depth by applying a second gating (as shown later) and 12 detecting the position of peak values of UT reflection in this region. The second gating is placed 13 between the front and back wall UT reflections of the composite sample. In this way, the first gating 14 provides an effective way to reduce the influences of noises on the conversion to C-Scan images because of the high signal-to-noise ratio (SNR) in the selected region, and the defect centre (X and Y index) can be located accurately in the C-Scan image afterwards. Moreover the UT reflection is the strongest at the central position of the defect (i.e. high SNR), the peak detection in the second gating region can give an accurate estimation of the defect depth.

19 In the experiment, raw A-Scan data was first acquired for each scanning position during a single scan.

20 The first gating in the double gating strategy was then applied to produce the corresponding C-Scan 21 image for this single scan. Taking the defect column with a depth of $0.2 \mathrm{~mm}$ as an example is shown in Fig. 7 to demonstrate the generation process of C-Scan image from A-Scan data, which indicate the size of each defect and the distance between them. The A-Scan data at a scan position with and without defect is shown against the time scale in Fig. 8. The resultant C-Scan image produced using 25 the gating and possible defects are marked by the blue or green colour shown in Fig. 8. Please note that Fig. 8 is a $1 \mathrm{DC}$ SeanB-Scan image due to the $1 \mathrm{D}$ decoder used in the manual scan for the curved

27 GFRP sample, which is also true for the following Figs. 9 11. The first possible defect on the left of 
1 the C-Scan image in Fig. 8, however, is false since no defect appears at this position in Fig. 7. Except

2 the smallest defect with a diameter of $4 \mathrm{~mm}$, other three defects are clearly spotted in the C-Scan

3 image with good contrast to their surroundings. The possible reason for missing the smallest defect

4 would be that it only induces a very small change in the UT reflection which is in the same level as

5 the noise. To segment the defects from the background in the C-Scan image, automated thresholding

6 techniques were applied. Prior to thresholding, a median filter was used to reduce the noises in the C-

7 Scan image, the window size of which was varied for optimised performance. The segmentation

8 results using entropy-based thresholding and a median filter with a window size of 3 or 5 or 7 or 9

9 pixels to evaluate the effect of the window size are shown in Fig. 9 (b) (e) respectively. The result

10 without filtering is also shown in Fig. 9 (a) for comparison. By applying the criterion that no false

11 defect should be produced in the segmented image, as many as possible defects should be identified

12 and the defect sizes should be as close as possible to their true values, the window size of 7 pixels is

13 found to be the optimal. The smallest defect, however, was missed in the segmented image. Thus

14 different thresholding techniques were applied, among which the moments-based method and

15 minimum error algorithm can give a similar result as the entropy-based method. The Otsu's method was also applied for thresholding and the results with pre-filtering or post morphological operation are

17 shown in Fig. 10. Note that the window size of 7 pixels for the median filter was also the best option

18 (with size accuracy of up to $3 \mathrm{~mm}$ ). It is shown that the segmented defect sizes by Ostu's thresholding

19 are less accurate and mostly larger than those by entropy-based thresholding, except for the smallest

20 defect, since a lower threshold was obtained in this case. Nevertheless, Ostu's thresholding is better

21 than entropy-based thresholding in the sense that it can identify all the four defects in the GFRP

22 sample.

23 As described above, the longitudinal size of each defect can be determined after applying appropriate thresholding techniques to the UT C-Scan image. The same method can be used for estimating the 2D

25 size of a defect if a 2D UT scanning is involved. With the binary image after thresholding, it is also 26 possible to determine the geometry centre of each segmented defect. The A-Scan data at this 27 geometry centre can be re-examined to derive the depth of the defect by applying a second gating as 
1 discussed previously. This gating lies between the UT reflections from the front and back wall of the

2 composite sample. Because in the above example, the four defects have a depth of $0.2 \mathrm{~mm}$, i.e. the

3 defects are very close to the front wall of the composite sample. This results in that the UT reflections

4 from the defects merge into the reflection from the front wall. It poses challenges to separate these

5 two signals and thus estimating the defect depth is not accurate. Another example is given regarding

6 the four defects with a depth of $1.4 \mathrm{~mm}$ as mentioned above. The same gating as shown in Fig. 8 was

7 applied to generate the C-Scan image from the corresponding A-Scan data. The entropy-based

8 thresholding was also adopted to segment the defects from the background in the C-Scan image, the

9 result of which is shown in the top right of Fig. 11. Note that the largest defect of $10 \mathrm{~mm}$ in diameter

10 among the four defects, which is located on the most right of the sample, was not scanned in the

11 experiment due to the length limit of the encoder. The other three defects, however, are clearly

12 identified in Fig. 11. The centre of each defect in Fig. 11 can be obtained using image processing

13 techniques in term of $\mathrm{x}$ and $\mathrm{y}$ indexes. Those two indexes can then be utilized to find the

14 corresponding raw A-Scan data at this scan position. This process can be made automated. Fig. 11

15 also shows two sets of A-Scan data for comparison with one at the centre of the defect marked by the green circle in the C-Scan image and another at a defect-free position close to the defect. In Fig. 11, it

17 is obvious that the UT reflection of the back wall of the composite sample is much lower at the defect

18 centre than at the defect-free position due to the attenuation caused by the defect. A peak appears

19 between the front and back wall reflections at the defect centre, the location of which tells the defect

20 depth. An appropriate second gating is applied to detect the peak location and then estimate the defect

21 depth, as shown in Fig. 11. Note that the depth in the $\mathrm{X}$ axis is calculated directly by multiplying the

22 ultrasonic speed in GFRP material with the arrival time of the UT reflection signal. Since UT

23 reflection signal transmits twice the distance between the reflection interface and the probe, this depth

24 is the twice of the real depth from the UT probe. The depth of the defect marked with the green ellipse

25 from the front wall of the composite sample was derived to be $1.29 \mathrm{~mm}$, i.e. (X index of peak -

$262 \times$ Wedge thickness $) / 2=(22.58-2 \times 10) / 2=1.29 \mathrm{~mm}$, which is slightly smaller than the real depth of

$271.4 \mathrm{~mm}$. For the other two defects on the left of the marked defect in Fig. 11, the same method was 
1 used to derive their depth. The estimated depths are 1.35 and $1.29 \mathrm{~mm}$, respectively, which are also

2 close to the real depth of $1.4 \mathrm{~mm}$ (i.e. largest difference is $0.11 \mathrm{~mm}$ ).

3 4.2. Processing results for flat CFRP sample

\section{$4 \quad$ 4.2.1. Image processing with global gating}

5 The flat CFRP sample is scanned in an automated manner using the water immersion setup. With the

6 acquired A-Scan data, a C-Scan image can be generated using the double gating technique which is

7 shown in Fig. 12 (a). In the C-Scan image, some of the defects are obvious (dark blue colour) but 8 others are overwhelmed by noises. If no pre-filtering is performed, the thresholding result based on 9 the Otsu's method is shown in Fig. 12 (b) which prove very difficult to tell defects from noises. To 10 improve this result, median and Gaussian filters are applied prior to the thresholding and the resultant 11 binary images are shown in Fig. 12 (c) and 12 (d), respectively. In Fig. 12 (c) and 12 (d), defects can 12 be easily recognised with noises significantly reduced and it is shown that Gaussian low-pass filter is 13 slightly better with fewer artefacts. However, only larger-size defects are shown and smaller-size 14 defects are lost. Based on the thresholding results, the depth positions of those identified defects in the 15 CFRP sample can be estimated using the method as described above for the GFRP sample. The 16 obtained depths of the defects are $1.44 \mathrm{~mm}$ for the second layer of defects and $2.43 \mathrm{~mm}$ for the third 17 layer of defects, respectively. The depth of the first and fourth layer of defects cannot be estimated

\subsubsection{Image processing with successive local gating}

As shown, the processing results for the flat CFRP sample are not as good as the curved GFRP sample. One important reason would be the use of different UT scanning setups. Another reason would be the different structures and material properties of the two composites. In the experiment, it is observed that the A-Scan data is noisier for the GFRP sample than for the CFRP sample, especially the data

25 fraction between the front and back wall reflections. Scarponi and Briotti [12] also reported that 
1 technique is applied for defect identification in the GFRP sample. For CFRP, however, the gating can

2 also be put between the front and back wall reflections of the A-Scan data to generate C-Scan images,

3 as shown in Fig. 13 (a). A single global gating or multiple local gating (window-based) can be used,

4 but it was proved that the latter is better since the effect of noises can be supressed within a narrow

5 window. Multiple local gating can be applied successfully at different depths between the front and

6 back wall of the composite sample to identify the defects and estimate their depths. This can be

7 explained as defects at a depth with certain height would present in the C-Scan images with a local

8 gating at this particular depth and successive depths covering the defect height. Through image

9 thresholding, defects in each C-Scan image can be identified, which corresponds to a local depth interval. For the same defect, it would be identified in several C-Scan images with successive local gating, i.e. it corresponds to several successive depth intervals which can be added up to estimate the potentially enables approximate $3 \mathrm{D}$ imaging of the defects in a composite since all the information regarding the 3D positions, 3D shapes and sizes of the defects can now be obtained approximately by processing relevant $2 \mathrm{D}$ C-Scan images and a mapping from $2 \mathrm{D}$ to $3 \mathrm{D}$ can be made to fulfil our purpose with the prior knowledge about the 3D geometries of the composite under test. It is worth to note that if the delamination is sufficient large in height, no UT reflection can be received from the material below the delamination.

For the flat CFRP sample, multiple local gating is achieved by equally dividing the depth between the front and back wall of the test sample (excluding the two dead zones of UT inspection near the front and back wall) to define several windows. Note that the equal division of the inspection depth is applied to enable automated operation and the number of divisions can be determined in the calibration stage. Here, the number of windows is chosen to be five based on trial-and-observation, namely window A, B, C, D and E with the depth increasing. A C-Scan image is generated within each window through gating, followed by Gaussian low-pass filtering. Then thresholding is applied to each filtered C-Scan image to identify defects at the particular depth within the sample. With the window C, no defects appear in the C-Scan image. The C-Scan images with the windows A, B, D and E are 
1 shown in Fig. 13 (a), (c), (e), and (g) and the corresponding binary images after thresholding are

2 shown in Fig. 13 (b), (d), (f) and (h). Note that the entropy-based thresholding method is used here. It

3 is shown that most defects can be segmented from the image background, but some artefacts due to

4 noises still exist in some of the binary images, e.g. Fig. 13 (d), (f) and (h). In Fig. 13 (h), it is very

5 difficult to segment the defects through thresholding due to very stronger noises as seen in Fig. 13 (g).

6 Most of these strong noises are from the reflections of the metal blocks in the experiment setup for

7 holding the test sample in position during the inspection process, as shown in Fig. 4. Also because the

8 defects within the window $\mathrm{E}$ are deeper and close to the back wall of the sample, their echoes are

9 weak and difficult to be separated from the back wall reflection. Only two defects can be spotted on the C-Scan image in Fig. 13 (g) and only one of them is shown after thresholding in Fig. 13 (h). All the other defects are clearly shown in other binary images after thresholding with their shape and size the identified defects, especially those close to the sample surface, are approximately round in shape.

14 The depth of each defect can also be estimated simply by referring to the corresponding gating window, as shown in the caption under each subfigure of Fig. 13.

Because the noises in the C-Scan images in Fig. 13 (c) and (e) are not as strong as in Fig. 13 (g) and weaker than the useful signals induced by defects, different thresholding methods are applied to remove the residual noises after thresholding shown in Fig. 13 (d) and (f). As abovementioned, hybrid methods can be adopted for improved thresholding performance, based on Huang's method [32], entropy-based thresholding and inter-modes method [33]. Huang's thresholding defines an index of fuzziness by measuring the distance between the grey-level image and its binary one. The optimal threshold is obtained by minimizing the index of fuzziness. Entropy based thresholding divides the histogram of an image into two probability distributions, representing the objects and background, respectively. The threshold is chosen such that the sum of the entropies of these probability distributions is maximized. Inter-modes method assumes a bimodal histogram and smooths the image histogram using a three-point mean filter iteratively until it has only two peaks (local maxima). The threshold is then set as the mean of these two local maxima. With successive local gating, it was 
1 found that the histogram of C-Scan images after Gaussian filtering has two extremely unequal peaks.

2 The much smaller peak is very close to zero, and the region around the larger peak is mainly

3 composed of noise-contaminated grey-levels which are close to those of defects, causing difficulties

4 in thresholding. This histogram does not confirm to the bimodal assumption in inter-modes method, and a slightly larger threshold is given. In principle, With the specificity of this image histogram, inter-modes method or entropy-based thresholding would derive the smallest a threshold between the two peaks of histogram (around the much larger peak due to its larger population of pixels), while Huang's method would produce the-a largest-much larger ene-threshold to minimize the index of fuzziness (i.e. the distance between the grey-level image and its binary onemuch larger than the other two). Based on our data, Trial and-errorempirical result shows that the average of entropy-based threshold and one of the other two would give an appropriate threshold with reduced noises. With

12 these findings, Huang's method is applied to the filtered C-Scan images in Fig. 13 (c), with the binary 13 image shown in Fig. 14 (a). In Fig. 14 (a), it is shown that the residual noises are removed but the smallest defect is missing, which is clearly shown in Fig. 13 (d). This means that the thresholds calculated by these two methods are either smaller or larger than the optimal threshold, i.e. these two methods are complementary to each other. The average of these two thresholds is then used, resulting in a better binary image as shown in Fig. 14 (b). Inter-modes method is applied to the filtered C-Scan image in Fig. 13 (e) and the binary image is shown in Fig. 15 (a) with residual noises removed and all defects remained. The average threshold of these two is also applied with the result shown in Fig. 15 (b).

\subsection{Discussion}

For manual inspection of the curved GFRP sample, 1D-C-SeanB-Scan images can be produced by applying the first gating in a double gating scheme. A window size of 7 pixels was proved to be optimal for the 1D median filtering before thresholding. It is shown that Otsu's thresholding outperforms other three methods (entropy-based, moments-based and minimum error thresholding) combined with median filtering in the sense that it can detect all the four defects. Regarding the accuracy of defect sizing, entropy-based thresholding (or moments-based or minimum error 
1 thresholding) can give a better result, which derives a larger threshold for image segmentation than

2 Otsu's method. By applying the second gating and signal peak detection after localization of the

3 defect centre, the depths of defects in the sample can be obtained, except for those close to the front

4 and back wall of the sample. It is shown that the estimated depths are close to their real value with a

5 maximum deviation of $0.11 \mathrm{~mm}$ for the second-layer defects in the sample.

6 For automated inspection of the flat CFRP sample, 2D C-Scan images can be generated either by

7 global gating mentioned above or successive local gating. With global gating, it is shown that image

8 filtering before thresholding is necessary and Otsu's thresholding was combined with Gaussian low-

9 pass or 2D median filtering for image segmentation with the former producing better results in the

10 sense of fewer artefacts. Defect depth can also be estimated using the method proposed for the GFRP

11 sample and larger estimation error is present for deeper defects, i.e. the third layer defects in the

12 CFRP sample. With successive local gating, C-Scan images with improved contrast and reduced noises can be obtained and Gaussian low-pass filter can be applied to further reduce the noises.

14 Entropy-based method and Huang's thresholding were found to be complementary regarding image 15 segmentation, as well as entropy-based method and inter-modes thresholding, which can be combined to be hybrid methods for image thresholding. The hybrid method can give better segmentation results

17 with minimal artefacts. It is shown that the proposed hybrid method with successive local gating can identify more defects, i.e. 13 out of 16 , than the global gating, i.e. 7 out of 16 , with the defect depth readily estimated by referring to the corresponding local gating window. With the successive local gating, approximate 3D imaging is also possible.

21 Based on the above discussion, the procedure for automated defect detection with automated UT scan 22 can be conducted as follows: (1) calibration stage: choose global gating or set the number of 23 successive local gating for C-Scan image generation, and the window size of image filter (e.g. 24 Gaussian low-pass filter) by pre-test; (2) automated detection of the front and back wall positions of 25 the sample in the A-Scan data at each scan point, and attain the filtered C-Scan images based on the 26 parameters set on step (1); (3) calculate thresholds of the C-Scan images using a variety of different 27 methods, take the average of each possible pair of complementary methods to segment the filtered C- 
1 Scan images, and fuse the obtained binary images into to one based on pixel-wise majority-vote

2 method; (4) automated morphological post-filtering of the binary image to remove small residual

3 spots due to noises based on the required detection resolution; (5) determine the position and size of

4 the identified defects in the binary image. In this procedure, each step can be accomplished in an

5 automated manner. The majority-vote method can improve the robustness of defect detection.

\section{5. Conclusions}

7

8 This paper presents a defect recognition technique for GFRP and CFRP composites based on image

9 processing. Artificial defects, with varied size and depth, were made and embedded into laminates to

10 simulate delamination in a curved GFRP sample and a flat CFRP sample for test. By using

11 appropriately chosen gating schemes, image filters and thresholding methods, all the 4 defects in a 1D manual scan can be detected for the curved GFRP sample and 13 out of 16 defects can be detected for the CFRP sample, with their 3D locations, sizes and sometimes shapes identified. Different thresholding algorithms were compared regarding defect segmentation and some of them were combined for improved performance. It is concluded that the proposed UT inspection technique can enhance the probabilities of detection for artificial delamination in composite materials. It also has a potential to be used for approximate 3D ultrasonic imaging of defects in composites. This paper, however, is only a feasibility study. Further investigation and more practical experimental validations are still needed. To enable completely automated defect recognition, more efforts have to be made regarding the UT scanning with appropriate coupling between the probe and sample and robust image processing and defect recognition. References regarding the local thicknesses of 'healthy' composites are also required for delamination detection when they are varied along the composites since it is difficult to distinguish large-sized delamination and back wall of the material from UT reflection signals. In practical applications, however, only large-sized delamination (more than $6 \mathrm{~mm}$, namely) are the detection target since they can cause serious mechanical failures and also the estimation of their sizes does not need to be very accurate with their locations accurately estimated. In this sense, the proposed image processing technique can be applied with satisfactory results. It is revealed that 
1 this automated system can offer enhanced defect detection probabilities and characterization

2 capabilities. This can potentially enable the effective control of the composite manufacturing process,

3 resulting in an increase in production yield and detection possibility of early-stage in-service defects

$4 \quad$ and a decrease in manufacturing and maintenance costs.

5

\section{6. Acknowledgements}

7 The research leading to these results has received funding from the UK's innovation agency, Innovate

8 UK under grant agreement No $\mathbf{1 0 1 8 0 0}$. The research has been undertaken as a part of the project

9 entitled "Automated ultrasonic inspection of aerospace composites with enhanced defect detection 10 probabilities aided by gantry deployed, CAD controlled robotics". The AutoDISC project is a 11 collaboration between the following organisations: Plant Integrity Ltd, Brunel Innovation Centre, 12 Kingston Computer Consultancy Ltd, Innovative Technology \& Science Ltd, Jackweld Ltd and 13 NetComposites.

\section{References}

17 [1] http://www.airbus.com/aircraftfamilies/passengeraircraft/a350xwbfamily/technology-andinnovation/, last accessed on 23/08/2017.

[2] E.A. Birt, R.A. Smith. Insight, 46: 681-686 (2004).

[3] A.S. Birks, P. McIntire, R.E. Green. ASNT Non-destructive testing handbook, Volume 7: Ultrasonic testing, 2nd Ed., p. 197-244. American Society for Non-destructive Testing, American Society for Metals (1991).

[4] B. Hayman, C. Berggreen, N.G. Tsouvalis. Proc. of the 1st International Conference on Marine Structures, Glasgow, UK (2007).

25 [5] T. Hasiotis, E. Badogiannis, N.G. Tsouvalis. J. Mech. Eng., 57: 192-203 (2011). UNESCO-EOLSS, Sample Chapter, p. 103-143 (2009). 
1 [7] M.R.P.D. Rao. Review of non-destructive evaluation techniques for FRP composite structural components, Master dissertation, West Virginia University, USA (2007).

3 [8] P.A. Lloyd. Ultrasonics. 27: 8-18 (1989).

4 [9] K. Imielinska, M. Castaings, R. Wojtyra, J. Haras, E. Le Clezio, B. Hosten. J Mater Process Tech 5 157-158: 513-522 (2004).

6 [10] L. Liu, B.M. Zhang, D.F. Wang, Z.J. Wu. Compos. Struct. 73: 303-309 (2006).

7 [11] J. Chang, C. Zheng, Q-Q Ni. Compos. Struct. 75: 451-456 (2006).

8 [12] C. Scarponi, G. Briotti. Compos Part B-Eng. 31: 237-243 (2000).

9 [13] B.R. Tittmann and R.L. Crane. Comprehensive Composite Materials. Volume 5, Ultrasonic 10 inspection of composites, p. 259-320, Elsevier, New York (2000).

11 [14] Z. Bergant, J. Janez, J. Grum. Proc. of the 12th International Conference of the Slovenian Society 12 for Non-Destructive Testing, Portorož, Slovenia (2013).

13 [15] R.A. Smith, L.J. Nelson, M.J. Mienczakowski, R.E. Challis. Insight. 51: 82-87 (2009).

14 [16] A. Ragondet. Experimental characterisation of the vacuum infusion process, PhD thesis. 15 University of Nottingham. UK (2005).

16 [17] http://www.zetec.com/topaz/, last accessed on 23/08/2017.

17 [18] P. Soille. Morphological Image Analysis: Principles and Applications, Springer, New York $18 \quad(2003)$.

19 [19] R.C. Gonzalez, R.E. Woods. Digital Image Processing, Pearson Education, Second Edition $20 \quad$ (2005).

21 [20] J. Yao, Image processing in tumor imaging, New techniques in oncologic imaging p: 79-102 22 (2006).

23 [21] K. Wong, Medical image segmentation: methods and applications in functional imaging, 24 Handbook Biomed. Image Anal. Segmentation Models Part B, 2:111-182 (2005).

25 [22] A. Farag, M. Ahmed, A. El-Baz, H. Hassan, Advanced segmentation techniques. Handbook of 26 biomedical image analysis I: segmentation models part A., Springer US, p: 479-533 (2005).

27 [23] C. Xu, D. Pham, J. Prince, Current methods in medical image segmentation, Ann. Rev. Biomed. 28 Eng. Ann. Rev., 2:315-337 (2000). 
1

2

3

4

5

6

7

8

9

10

1 [24] H. Zaidi, I. El-Naqa, Eur. J. Nucl. Med. Mol. Imaging, 37: 2165-87(2010).

2 [25] M. Sezgin, B. Sankur. J. Electron. Imaging. 13(1): 146-165 (2004).

3 [26] A.K.C. Wong, P.K. Sahoo, S. Soltani, Y.C. Chen. Comput. Vision Graph. 41: 233-260 (1988).

4 [27] W. Tsai. Comput. Vision Graph. 29: 377-393 (1985).

5 [28] J.N. Kapur, P.K. Sahoo, and A.K.C. Wong. Comput. Vision Graph. 29: 273-285 (1985).

6 [29] N. Otsu. IEEE Trans. Systems Man Cybernet. 9: 62-66 (1979).

7 [30] J. Kittler, J. Illingworth. Pattern. Recogn. 19: 41-47 (1986).

8 [31] R.M. Haralick, S.R. Sternberg, X. Zhuang. IEEE T Pattern. Anal.; 9(4): 532-550 (1987).

9 [32] L.K. Huang, M.J.J. Wang. Pattern. Recogn. 28: 41-51 (1995).

10 [33] C.A. Glasbey. Graph. Model. Im. Proc. 55: 532-537 (1993). 

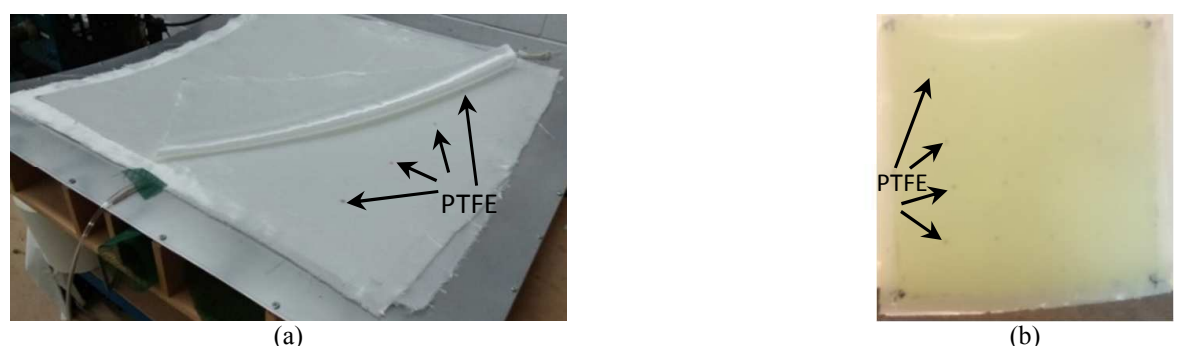

Fig. 1. Defect distribution in curved GFRP sample. (a) Lay up of GFRP laminate layers with simulated defects embedded and (b) Manufactured GFRP sample with PTFE defects.

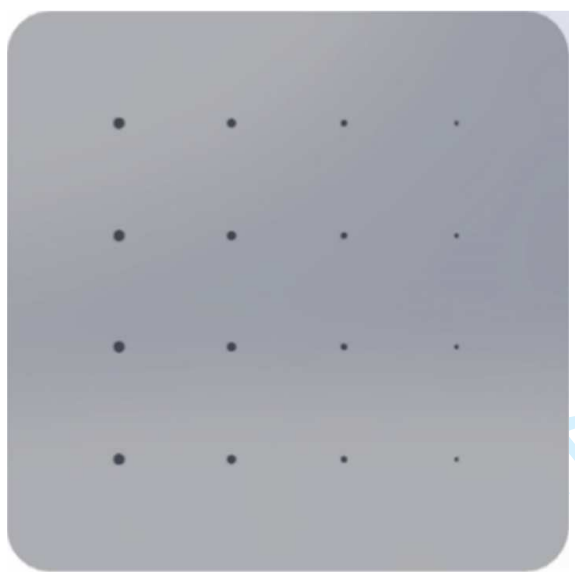

Fig. 2. Modelled defect distribution in flat CFRP sample.

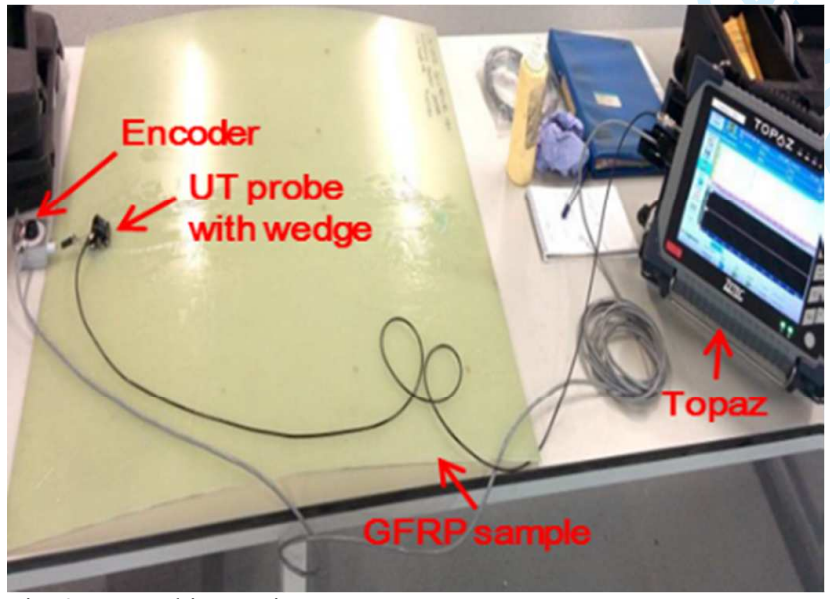

Fig. 3. Manual inspection setup. 


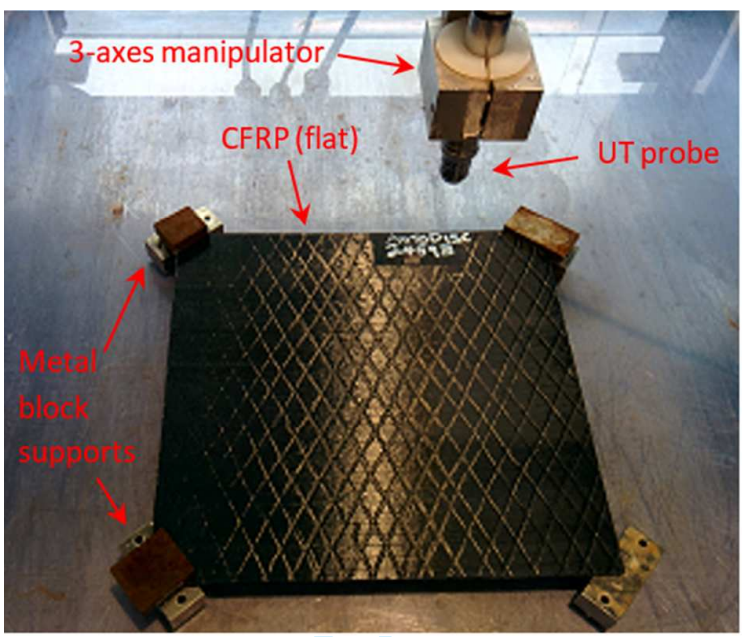

Fig. 4. Automated inspection setup.

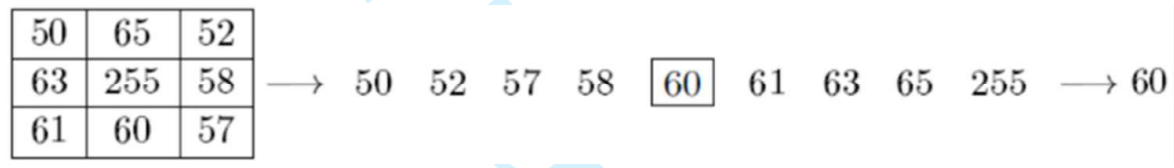

Fig. 5. Functional illustration of a typical 2D median filter.

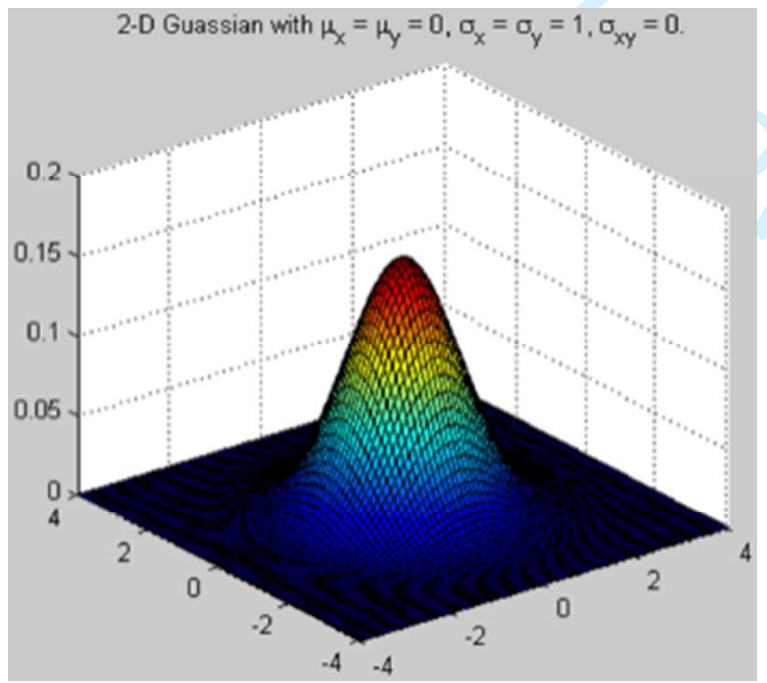

Fig. 6. Graphical representation of a typical 2D Gaussian distribution. 


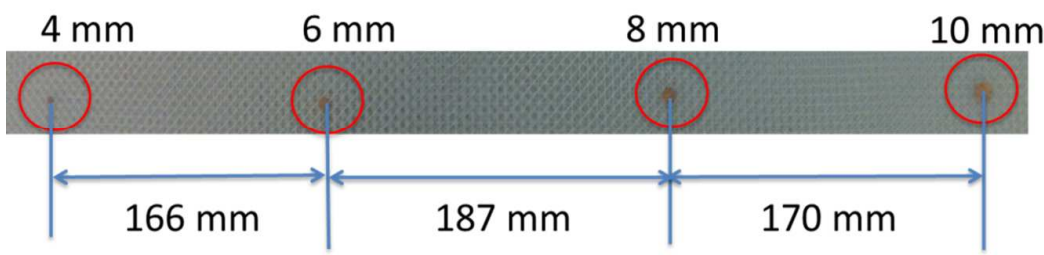

Fig. 7. Photo of a defect column with a depth of $0.2 \mathrm{~mm}$.

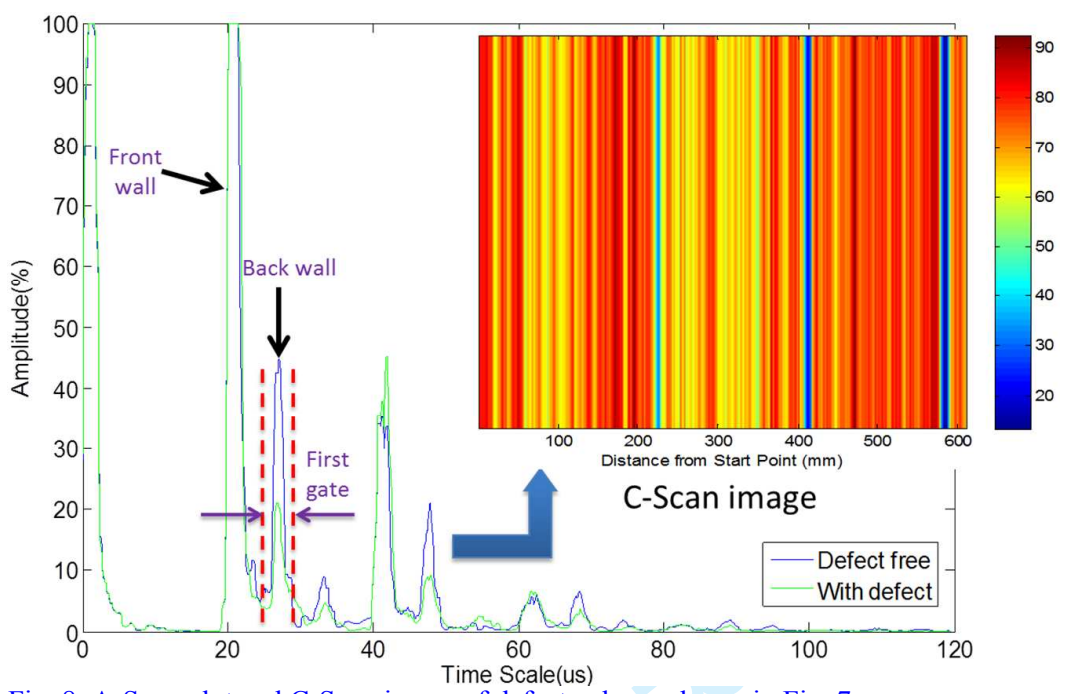

Fig. 8. A-Scan plot and C-Scan image of defect column shown in Fig. 7.

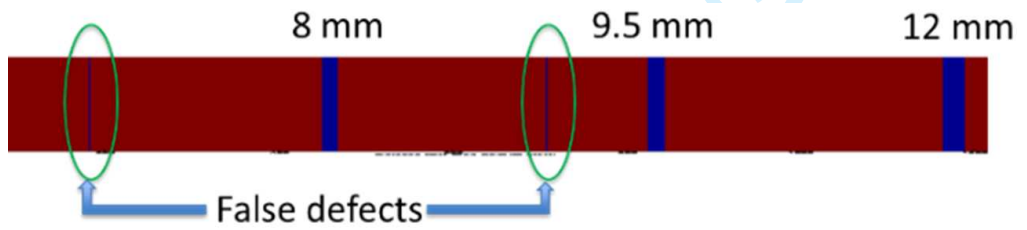

(a) Without filtering

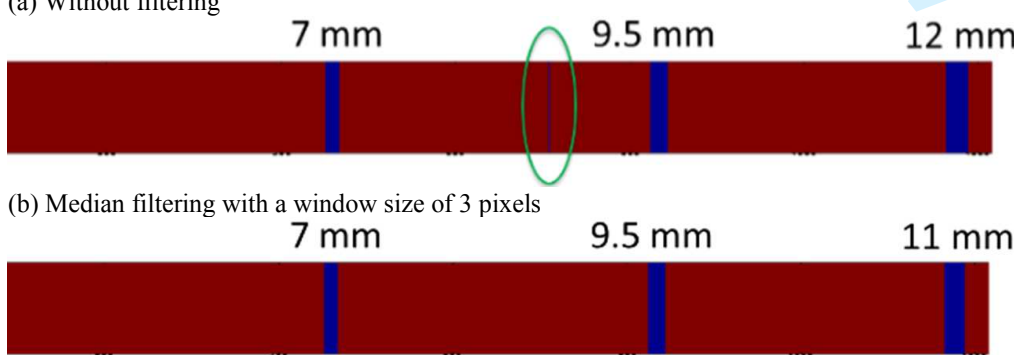

(c) Median filtering with a window size of 5 pixels
$6 \mathrm{~mm}$
$9 \mathrm{~mm}$
$10.5 \mathrm{~mm}$

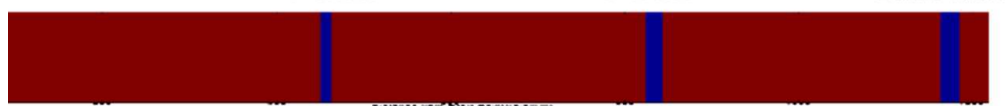

(d) Median filtering with a window size of 7 pixels

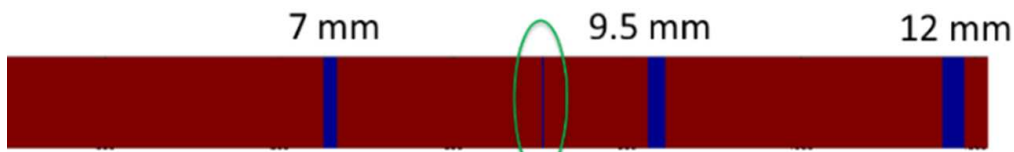

(e) Median filtering with a window size of 9 pixels

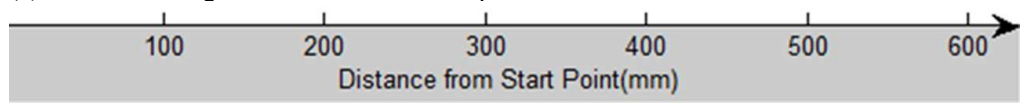


(f) $\mathrm{X}$ axis of subfigures (a) (e)

Fig. 9. Thresholding results using entropy-based method for curved GFRP

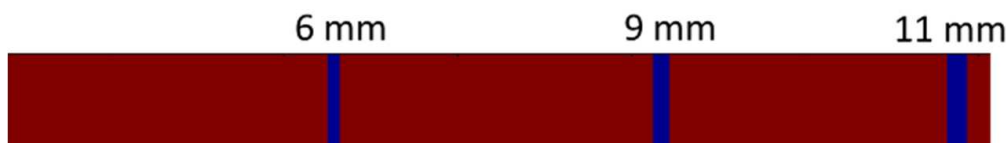

(a) Median filtering with a window size of 3 pixels

$$
6 \mathrm{~mm} \quad 9 \mathrm{~mm} \quad 10.5 \mathrm{~mm}
$$

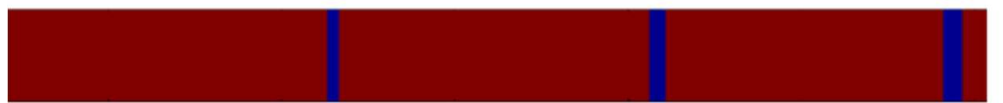

(b) Median filtering with a window size of 5 pixels

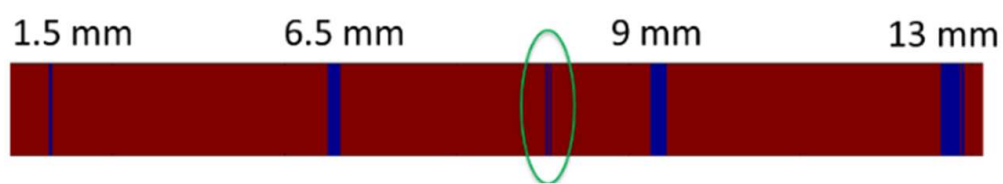

(c) Median filtering with a window size of 7 pixels

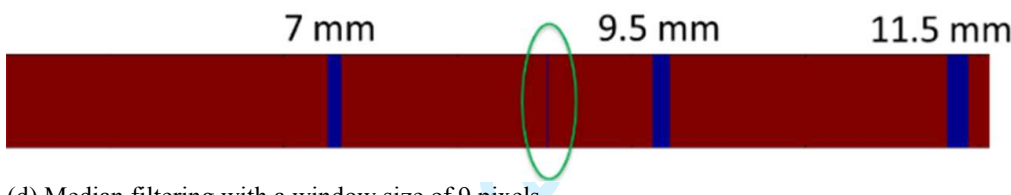

(d) Median filtering with a window size of 9 pixels

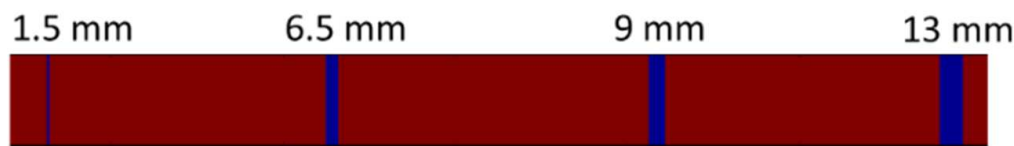

(e) Median filtering with a window size of 7 pixels and after morphological operation

\begin{tabular}{cccccc}
1 & 1 & 1 & 1 & 1 \\
\hline 100 & 200 & 300 & 400 & 500 & 600
\end{tabular}

(f) $\mathrm{X}$ axis of subfigures (a) (e)

Fig. 10. Thresholding results using Ostu's method for curved GFRP.

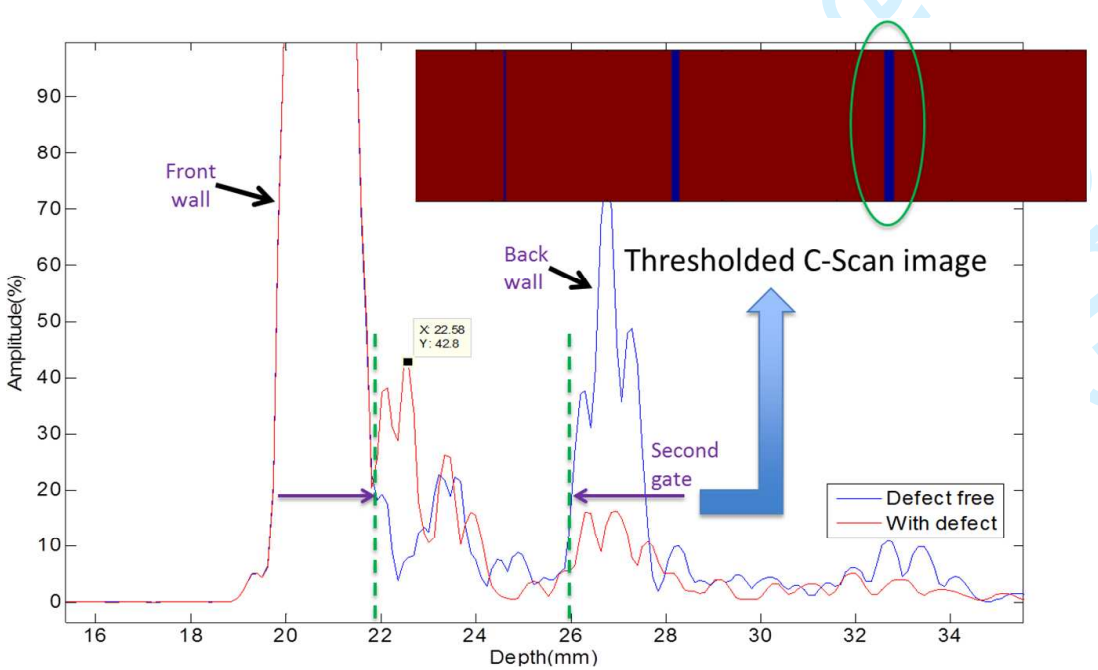

Fig. 11. Defect depth estimation by applying a second gating. 


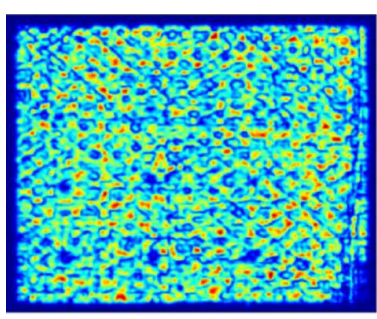

(a) C-Scan image

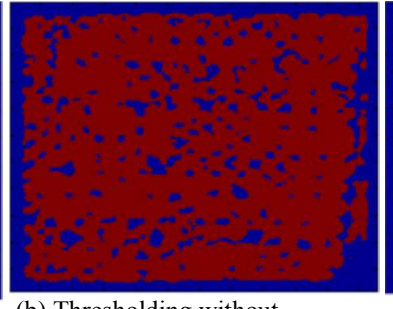

(b) Thresholding without filtering

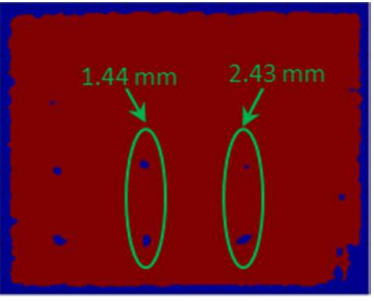

(c) With Gaussian filtering

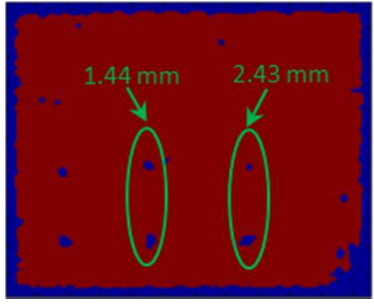

(d) With median filtering

Fig. 12. C-Scan imaging using global gating and thresholding results using Otsu's method for flat CFRP

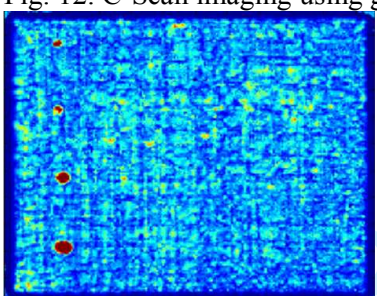

(a) C-Scan image in local window A (depth $0.6 \sim 1.14 \mathrm{~mm})$

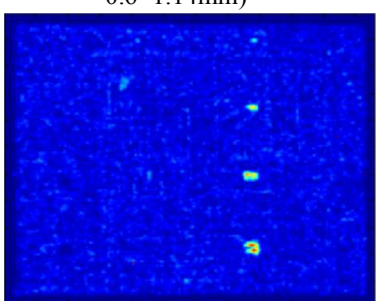

(e) C-Scan image in local window D (depth: $2.22 \sim 2.76 \mathrm{~mm})$

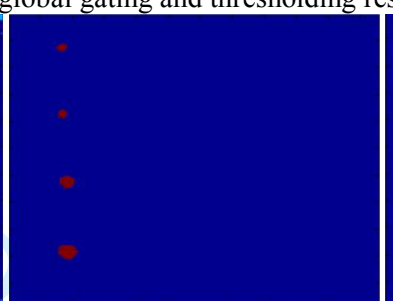

(b) Thresholding in local window A (depth: $0.6 \sim 1.14 \mathrm{~mm})$

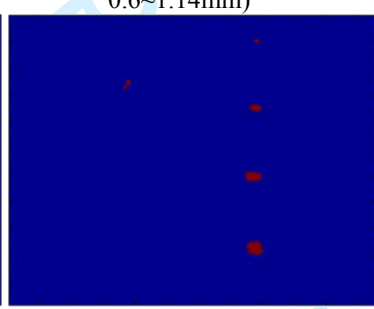

(f) Thresholding in local window D (depth:

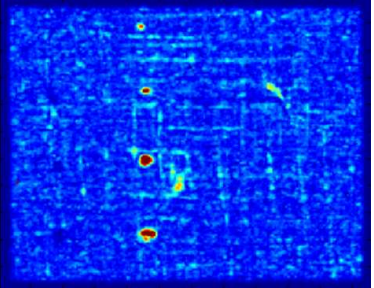

(c) C-Scan image in local window B (depth: $1.14 \sim 1.68 \mathrm{~mm})$

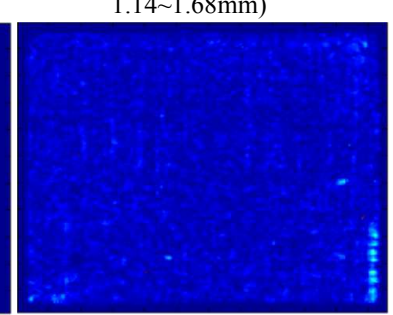

(g) C-Scan image in local window $\mathrm{E}$ (depth: $2.76 \sim 3.3 \mathrm{~mm}$ )

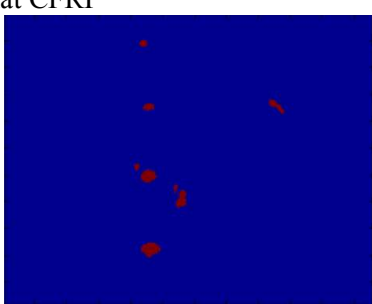

(d) Thresholding in local window B (depth $1.14 \sim 1.68 \mathrm{~mm})$

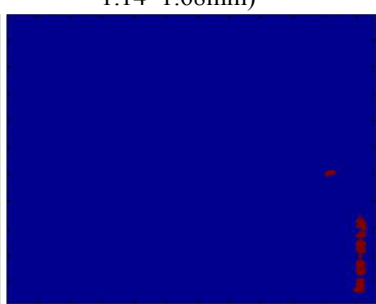

(h) Thresholding in local window $\mathrm{E}$ (depth: $2.76 \sim 3.3 \mathrm{~mm})$

Fig. 13. C-Scan imaging using local gating and thresholding using entropy-based method for flat CFRP.

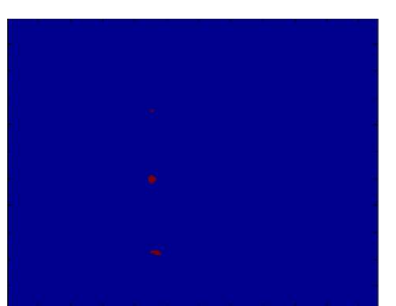

(a) Huang's thresholding (depth: $1.14 \sim 1.68 \mathrm{~mm}$ )

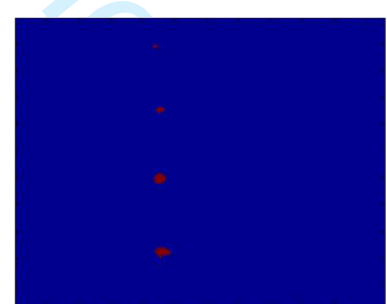

(b) Combined thresholding (depth: 1.14 1.68mm)

Fig. 14. Huang's thresholding and combined Huang's and entropy-based thresholding results in local window B.

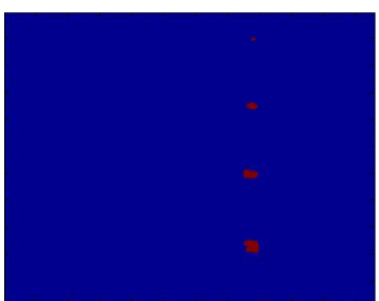

(a) Inter-modes thresholding (depth: 2.22 2.76mm)

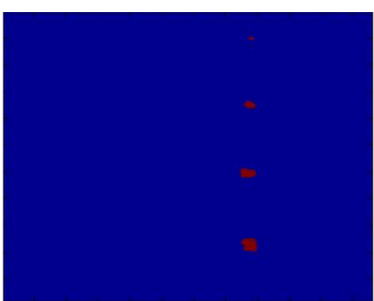

(b) Combined thresholding (depth: $2.22 \sim 2.76 \mathrm{~mm}$ )

Fig. 15. Inter-modes thresholding and combined Inter-modes and entropy-based thresholding results in local window D. 
Table 1 Properties of composite for testing (curved GFRP plate with PTFE defects)

\begin{tabular}{cc}
\hline Sample shape & Single-curved surface \\
\hline Dimensions, $l \times w \times d(\mathrm{~mm})$ & $850 \times 850 \times 3.6$ \\
Reinforcement & E-glass \\
Matrix & Epoxy infusion resin \\
Fabric architecture & linear density \pm 45 biaxial, $300 \mathrm{gsm}$ \\
Lay-up & 16 layers, $[0,90 / \pm 45] \mathrm{s}$ \\
Diameter of defects $(\mathrm{mm})$ & $4,6,8,10$ \\
Depth of defects $(\mathrm{mm})$ & $0.2,1.4,2.5,3.4$ \\
\hline
\end{tabular}

Table 2 Characteristics of simulated defects in flat CFRP sample

\begin{tabular}{lcccc}
\hline & Column 1 & Column 2 & Column 3 & Column 4 \\
\hline Row 1 & $4 \mathrm{~mm}, 0.3 \mathrm{~mm}$ & $4 \mathrm{~mm}, 1.5 \mathrm{~mm}$ & $4 \mathrm{~mm}, 2.7 \mathrm{~mm}$ & $4 \mathrm{~mm}, 3.3 \mathrm{~mm}$ \\
Row 2 & $6 \mathrm{~mm}, 0.3 \mathrm{~mm}$ & $6 \mathrm{~mm}, 1.5 \mathrm{~mm}$ & $6 \mathrm{~mm}, 2.7 \mathrm{~mm}$ & $6 \mathrm{~mm}, 3.3 \mathrm{~mm}$ \\
Row 3 & $8 \mathrm{~mm}, 0.3 \mathrm{~mm}$ & $8 \mathrm{~mm}, 1.5 \mathrm{~mm}$ & $8 \mathrm{~mm}, 2.7 \mathrm{~mm}$ & $8 \mathrm{~mm}, 3.3 \mathrm{~mm}$ \\
Row 4 & $10 \mathrm{~mm}, 0.3 \mathrm{~mm}$ & $10 \mathrm{~mm}, 1.5 \mathrm{~mm}$ & $10 \mathrm{~mm}, 2.7 \mathrm{~mm}$ & $10 \mathrm{~mm}, 3.3 \mathrm{~mm}$ \\
\hline
\end{tabular}

Table 3 Scan setting for manual inspection

\begin{tabular}{lccccccc}
\hline & $\begin{array}{c}\text { Start } \\
(\mathbf{m m})\end{array}$ & Quantity & $\begin{array}{c}\text { Resolution } \\
(\mathbf{m m})\end{array}$ & $\begin{array}{c}\text { Min } \\
(\mathbf{\%})\end{array}$ & $\begin{array}{c}\text { Max } \\
(\mathbf{\%})\end{array}$ & $\begin{array}{c}\text { Start } \\
(\%)\end{array}$ & $\begin{array}{c}\text { Resolution } \\
(\mathbf{\%})\end{array}$ \\
\hline $\begin{array}{l}\text { Scan Axis } \\
\text { Index }\end{array}$ & 0.00 & 1301 & 0.5 & - & - & - & - \\
Axis & 0.00 & 1 & 1.0 & - & - & - & - \\
UT Signal & 0.11 & 1092 & 0.03 & - & - & - & - \\
Amplitude & - & - & - & 0 & 100 & 0 & 0.002 \\
\hline
\end{tabular}

Table 4 Scan setting for automated inspection

\begin{tabular}{lccccccc}
\hline & $\begin{array}{c}\text { Start } \\
(\mathbf{m m})\end{array}$ & Quantity & $\begin{array}{c}\text { Resolution } \\
(\mathbf{m m})\end{array}$ & $\begin{array}{c}\text { Min } \\
(\mathbf{\%})\end{array}$ & $\begin{array}{c}\text { Max } \\
(\%)\end{array}$ & $\begin{array}{c}\text { Start } \\
(\%)\end{array}$ & $\begin{array}{c}\text { Resolution } \\
(\%)\end{array}$ \\
\hline $\begin{array}{l}\text { Scan Axis } \\
\text { Index }\end{array}$ & 0.00 & 231 & 1.0 & - & - & - & - \\
Axis & 0.00 & 206 & 1.0 & - & - & - & - \\
UT Signal & 75 & 1348 & 0.03 & - & - & - & - \\
Amplitude & - & - & - & 0 & 200 & 0 & 0.003 \\
\hline
\end{tabular}




\section{Response to reviewers' comments}

We would like to thank the reviewers again for their careful reading of the revised paper and for their constructive comments. We have revised the paper the second time according to their suggestions, and provided a detailed response below. In the revised manuscript, we have marked the changes.

\section{Reviewer \#1:}

I appreciate the revisions you made. I'd encourage you to either provide a citation for or further explain why Huang's method over estimates and the other two others underestimate the threshold. Also, I'd consider using a phrase that denotes a more systematic approach to determining how to merge multiple techniques than "trial-and-error" if it is appropriate to do so (pg 20, line 5).

Response: Thanks for the suggestions. Further explanation is given as follows: "With the specificity of this image histogram, inter-modes method or entropy-based thresholding would derive a threshold between the two peaks of histogram (around the much larger peak due to its larger population of pixels), while Huang's method would produce a much larger threshold to minimize the index of fuzziness (i.e. the distance between the grey-level image and its binary one). Based on our data, empirical result shows that the average of entropy-based threshold and one of the other two would give an appropriate threshold with reduced noises." These arguments have been added in the third paragraph of section 4.2.2 in the revised manuscript. The "trial-and-error" has been rephrased to be "empirical result" in the revised manuscript.

\section{Reviewer \#2:}

The authors have incorporated many changes in the revised manuscript. Attached are some minor suggestions/comments after which the manuscript can be accepted.

1. Table 3 and 4: what is UT sound?

Response: The "UT sound" in Table 3 and 4 has been corrected to be "UT signal" for clarity.

2. The term B-Scan is more appropriate than 1D C-Scan. Its universally accepted in the area of NDE.

Response: Thanks for the suggestion. The term 1D C-Scan has been changed to be B-Scan in the revised manuscript. 
Owners of U.S. government-funded works only - Please check the appropriate box and sign below.

$\square$ This is a Work of the federal government and is not subject to copyright.

$\square$ This is a Work for hire. The federal government retains the nonexclusive right to reproduce this work.

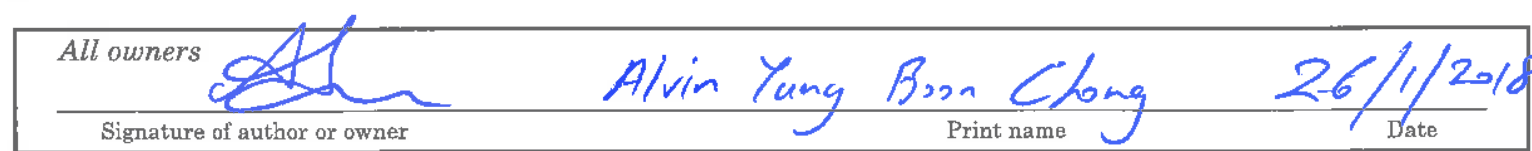

This document, fully executed, must be received before the manuscript can be accepted for publication. If the manuscript is not published by ASNT, this copyright transfer will not take effect. 
Owners of U.S. government-funded works only-Please check the appropriate box and sign below.

$\square$ This is a Work of the federal government and is not subject to copyright.

$\square$ This is a Work for hire. The federal government retains the nonexclusive right to reproduce this work.

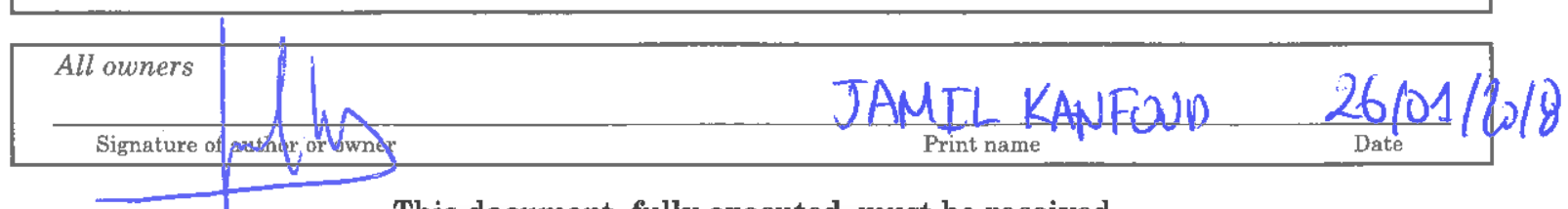

This document, fully executed, must be received before the manuscript can be accepted for publication. If the manuscript is not published by ASNT, this copyright transfer will not take effect. 
Owners of U.S. government-funded works only - Please check the appropriate box and sign below.

$\square$ This is a Work of the federal government and is not subject to copyright.

This is a Work for hire. The federal government retains the nonexclusive right to reproduce this work.

All owners $\mathrm{s}_{\text {Signature of author or owner }}$ Siamak Tavaksu

This document, fully executed, must be received before the manuscript can be accepted for publication. If the manuscript is not published by ASNT, this copyright transfer will not take effect. 


\section{Date: 2.5 Jonvery 2068}

The copyright, titie, interest, and all rights, including subsidiary and/or derivative rights, in all languages in the work titled:

" Automated quality characterization for composites using hybrid ultrasonic imaging techniques " ("work")

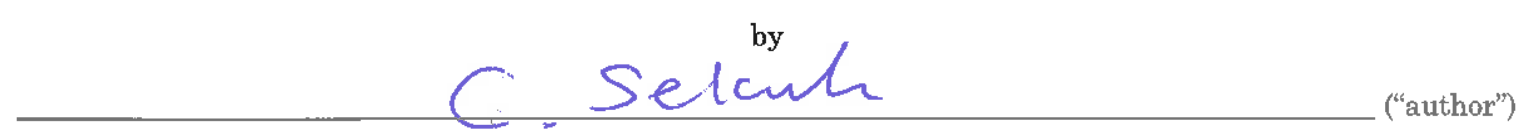

is hereby assigned and transferred by Brunel Innovation Centre (Brunel University London) ("owner") to The American Society for Nondestructive Testing, Inc. (hereinafter ASNT) effective if and when the work is accepted for publication. This copyright transfer is understood as including not only the text, but any integral part of the work, including but not limited to figures and tables. Furthermore, this assignment and transfer applies to all other publications or reproductions by ASNT or its licensees, in which the above work may be reproduced subsequent to its appearance in:

\section{Research in Nondestructive Evaluation}

ASNT reserves the right to edit this work. However, ASNT acknowledges that the following rights are not transferred hereunder:

1. Any proprietary rights (such as patent rights) other than copyright.

2. The right to make oral presentation of the same material in any form.

3. The right to grant or refuse permission to third parties to republish all or part of the work or translations thereof. Such third parties must obtain ASNT's written permission as well.

4. The right to use all or part of this work in future works of author in author's capacity as either author or editor, without charge, provided ASNT is properly credited as the source.

The owner represents and warrants that, to the best of his/her knowledge, the work does not contain material the publication of which would violate any copyright, trademark, intellectual property rights or other personal or proprietary rights of any private person or entity or governmental or quasi-governmental entity. The owner further affirms that written permission has been secured by any person depicted in any photograph submitted for publication. Caveat: the owner is specifically reminded and cautioned that NDT processes, procedures, techniques, etc. may be the subject of U.S. government classification regulations; the unauthorized publication thereof may expose the author and/or owner to prosecution under applicable federal law. Therefore, the owner must carefully verify sources of data other than those originated by the author. In addition, the owner warrants that the work has not been published previously elsewhere.

Owners of U.S. government-funded works only - Please check the appropriate box and sign below.

$\square$ This is a Work of the federal government and is not subject to copyright.

$\square$ This is a Work for hire. The federal government retains the nonexclusive right to reproduce this work.

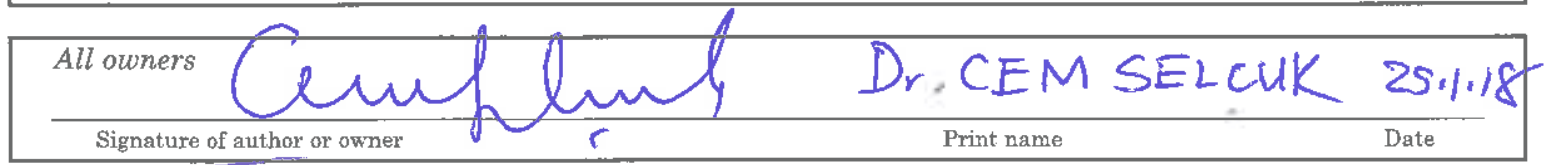

This document, fully executed, must be received before the manuscript can be accepted for publication. If the manuscript is not published by ASNT, this copyright transfer will not take effect. 
The copyright, title, interest, and all rights, including subsidiary and/or derivative rights, in all languages in the work titled:

\section{" Automated quality characterization for composites using hybrid ultrasonic imaging techniques " ("work")}

Jiangtao Sun

by ("author")

is hereby assigned and transferred by Brunel Innovation Centre (Brunel University London) ("owner") to The American Society for Nondestructive Testing, Inc. (hereinafter ASNT) effective if and when the work is accepted for publication. This copyright transfer is understood as including not only the text, but any integral part of the work, including but not limited to figures and tables. Furthermore, this assignment and transfer applies to all other publications or reproductions by ASNT or its licensees, in which the above work may be reproduced subsequent to its appearance in:

\section{Research in Nondestructive Evaluation}

ASNT reserves the right to edit this work. However, ASNT acknowledges that the following rights are not transferred hereunder:

1. Any proprietary rights (such as patent rights) other than copyright.

2. The right to make oral presentation of the same material in any form.

3. The right to grant or refuse permission to third parties to republish all or part of the work or translations thereof. Such third parties must obtain ASNT's written permission as well.

4. The right to use all or part of this work in future works of author in author's capacity as either author or editor, without charge, provided ASNT is properly credited as the source.

The owner represents and warrants that, to the best of his/her knowledge, the work does not contain material the publication of which would violate any copyright, trademark, intellectual property rights or other personal or proprietary rights of any private person or entity or governmental or quasi-governmental entity. The owner further affirms that written permission has been secured by any person depicted in any photograph submitted for publication. Caveat: the owner is specifically reminded and cautioned that NDT processes, procedures, techniques, etc. may be the subject of U.S. government classification regulations; the unauthorized publication thereof may expose the author and/or owner to prosecution under applicable federal law. Therefore, the owner must carefully verify sources of data other than those originated by the author. In addition, the owner warrants that the work has not been published previously elsewhere.

Owners of U.S. government-funded works only-Please check the appropriate box and sign below.

$\square$ This is a Work of the federal government and is not subject to copyright.

This is a Work for hire. The federal government retains the nonexclusive right to reproduce this work.

\begin{tabular}{|ccc|}
\hline All owners jiangfow Sun & JIANGTAO SUN & $26 / 1 / 2018$ \\
\hline Signature of author or owner & Print name & Date \\
\hline
\end{tabular}

This document, fully executed, must be received

before the manuscript can be accepted for publication. If the manuscript is not published by ASNT, this copyright transfer will not take effect. 
Date:

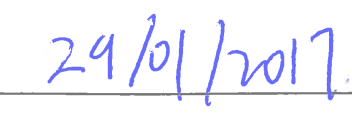

The copyright, title, interest, and all rights, including subsidiary and/or derivative rights, in all languages in the work titled:
“ Automated quality characterization for composites using hybrid ultrasonic imaging techniques " ("work") by
Guojin Feng ("author")

is hereby assigned and transferred by

Brunel Innovation Centre (Brunel University London) ("owner") to The American Society for Nondestructive Testing, Inc. (hereinafter ASNT) effective if and when the work is accepted for publication. This copyright transfer is understood as including not only the text, but any integral part of the work, including but not limited to figures and tables. Furthermore, this assignment and transfer applies to all other publications or reproductions by ASNT or its licensees, in which the above work may be reproduced subsequent to its appearance in:

\section{Research in Nondestructive Evaluation}

ASNT reserves the right to edit this work. However, ASNT acknowledges that the following rights are not transferred hereunder:

1. Any proprietary rights (such as patent rights) other than copyright.

2. The right to make oral presentation of the same material in any form.

3. The right to grant or refuse permission to third parties to republish all or part of the work or translations thereof. Such third parties must obtain ASNT's written permission as well.

4. The right to use all or part of this work in future works of author in author's capacity as either author or editor, without charge, provided ASNT is properly credited as the source.

The owner represents and warrants that, to the best of his/her knowledge, the work does not contain material the publication of which would violate any copyright, trademark, intellectual property rights or other personal or proprietary rights of any private person or entity or governmental or quasi-governmental entity. The owner further affirms that written permission has been secured by any person depicted in any photograph submitted for publication. Caveat: the owner is specifically reminded and cautioned that NDT processes, procedures, techniques, etc. may be the subject of U.S. government classification regulations; the unauthorized publication thereof may expose the author and/or owner to prosecution under applicable federal law. Therefore, the owner must carefully verify sources of data other than those originated by the author. In addition, the owner warrants that the work has not been published previously elsewhere.

Owners of U.S. government-funded works only - Please check the appropriate box and sign below.

$\square$ This is a Work of the federal government and is not subject to copyright.

$\square$ This is a Work for hire. The federal government retains the nonexclusive right to reproduce this work.

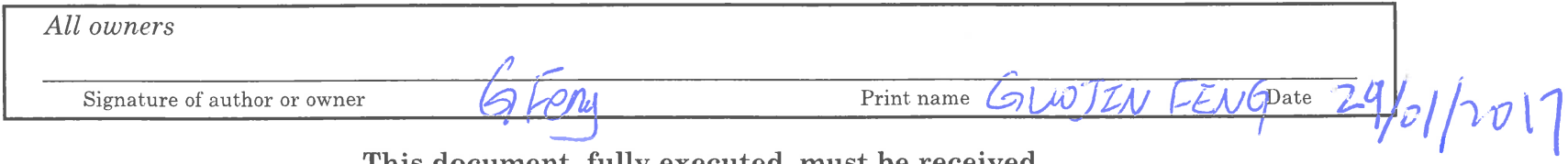

This document, fully executed, must be received

before the manuscript can be accepted for publication. If the manuscript is not published by ASNT, this copyright transfer will not take effect. 


\section{COPYRIGHT PROCEDURES}

Research in Nondestructive Evaluation is a journal owned by the American Society for Nondestructive Testing.

ASNT requires that all authors of an article complete a specific ASNT copyright. Each author must sign a copyright form, however all signatures do NOT have to be on the same form.

Please download and complete the copyright form (second page of this PDF) and send it to ASNT.

Forms can be scanned, saved as PDF or JPG and e-mailed to RNDECopyright@asnt.org 
Owners of U.S. government-funded works only - Please check the appropriate box and sign below.

$\square$ This is a Work of the federal government and is not subject to copyright.

$\square$ This is a Work for hire. The federal government retains the nonexclusive right to reproduce this work.

\begin{tabular}{|c|c|c|}
\hline All owners & T.H.GMN & $3 / 1 / 18$ \\
\hline Signature of author or phy & Print name & Date \\
\hline
\end{tabular}

This document, fully executed, must be received before the manuscript can be accepted for publication. If the manuscript is not published by ASNT, this copyright transfer will not take effect. 